Relations industrielles

Industrial Relations

\title{
Physiologie pratique du travail, par G. Lehman, Paris, Les
} Editions d'Organisation, 1955.

\section{R. Giroux}

Volume 15, numéro 1, janvier 1960

URI : https://id.erudit.org/iderudit/1022087ar

DOI : https://doi.org/10.7202/1022087ar

Aller au sommaire du numéro

Éditeur(s)

Département des relations industrielles de l'Université Laval

ISSN

0034-379X (imprimé)

1703-8138 (numérique)

Découvrir la revue

Citer ce compte rendu

Giroux, C. (1960). Compte rendu de [Physiologie pratique du travail, par G. Lehman, Paris, Les Editions d'Organisation, 1955.] Relations industrielles / Industrial Relations, 15(1), 149-150. https://doi.org/10.7202/1022087ar

Tous droits réservés (C Département des relations industrielles de l’Université Laval, 1960
Ce document est protégé par la loi sur le droit d'auteur. L’utilisation des services d'Érudit (y compris la reproduction) est assujettie à sa politique d'utilisation que vous pouvez consulter en ligne.

https://apropos.erudit.org/fr/usagers/politique-dutilisation/ 
vions établir des critères objectifs de performance, comme la quantité et la qualité de la production, aurions-nous besoin d'évaluer le rendement des employés?

Nous pourrions relever également bien d'autres points du texte qui prêtent à confusion et qui pourraient être interprétés de façon différente. Mais toutefois, n'oublions pas que ce texte ne s'adresse pas à des professionnels. Pour cela le style et le contenu se devaient d'être aussi simples que possible. La lecture en est très rapide.

Cet ouvrage intéressera les profanes, bien qu'il fasse de la psychologie appliquée une science trop facile à comprendre et à utiliser. Ne cherchez pas les références et la bibliographie, il n'y en a pas.

\section{R. GIRoux}

Physiologie pratique du travail, par G. Lehman, Paris, Les Editions d'Organisation, 1955.

Un travail de recherche exécuté par be directeur de l'Institut Max Plank de Dortmund devrait avoir une valeur scientifique exceptionnelle. Toutefois, il ne faut pas confondre l'Institut Max Plank de Dortmund, qui se spécialise en physiologie et en médecine, avec l'Institut Max Plank de Bohn, qui a formé de nombreux physiciens de renommée mondiale.

Lehman veut explorer dans les moindres détails les composantes physiologiques du travail humain. Selon lui, l'un des problèmes de la technologie humaine est de déterminer «les meilleures conditions d'harmonisation des gestes humains, de la pensée consciente et des actes de la machine ». Une telle entreprise nécessite une triple étude physiologique, psychologique et morale. Lehman s'est penché sur l'aspect physiologique du problème. Son approche est surtout inductive, partant de mouvements et de principes élémentaires. Dès le début, Lehman fit un exposé de la structure et de la fonction du muscle. De là il passe à l'étude de la coordination des mouvements et ensuite au comportement de la circulation sanguine au début et durant un travail corporel fatigant. Tout $y$ passe par la suite: le volume et les battements du coeur, la respiration, la consommation d'oxygène, etc. La fatigue $y$ est nécessairement étudiée, de même que l'influence des pauses sur la récupération. Plus loin l'auteur fait un bref exposé sur les positions du corps durant le travail, et il se lance immédiatement dans une étude approfondie du métabolisme au travail et au repos.

Cette recherche se continue ainsi sur une base physiologique pour arriver enfin au chapitre sur le développoment historique des outils. Lehman consacre plusieurs pages aux 12,000 pelles différentes fabriquées en Allomagne vers 1940. Il passe ensuite d la pioche, à la brouette et au < godendard \$. L'auteur termine avec quelques pages sur la température, le climat, le boire, etc.

Ce bref résumé du contenu de ce volume démontre clairement la méthode d'approche de Lehman. A partir de conclusions basées sur tres peu de matériel expérimental, l'auteur arrive à des principes empiriques qu'il essaie de confirmer par l'observation. L'approche de Lehman demeure très atomistique. En effet il va jusqu'a prendre des mesures plutôt simplistes, i.e. \& la mesure du volume du pied pour connaître la capacité à exécuter un travail debout (page 133). A ce compte, pourquoi ne pas mesurer la longueur de la langue de ceux qui font application pour une position de vendeur? C'est peut-être absurde, mais c'est la façon de procéder de Lehman dans bien des cas.

Néanmoins, il faut comprendre que les recherches de l'auteur furent complétées entre 1950 et 1951, puisque le présent ouvrage fut publié en 1952 et traduit en 1955. Depuis ce temps, il est évident que l'étude des relations homme-machine a progressé de façon bien différente aux Etats-Unis. Prenons comme exemple les travaux de Chapanis à l'Université Johns Hopkins. Combinant les méthodes de la psychologie expérimentale à celles du génio industriel, lui et ses collaborateurs ont formé une discipline nouvelle: le génie humain. Par exemple, ils dessinent un système de manettes et de cadrans ou d'autres appareils différents. Ensuite, de nombreux sujets sont exposés au système et essaient de résoudre les problèmes rencontrés. La phase sui- 
vante consiste à déterminer statistiquement les caractéristiques du système qui facilite la perception (interprétation des données sensorielles) et la performance. Cette méthode globale arrive beaucoup plus rapidement à des résultats pratiques que la méthode de Lehman, qui se rattache davantage à l'étude des scienoes pures. D'autre part, il faut admettre que Chapanis travaille à résoudre des problèmes immédiats qui sont définis dans les contrats obtenus des forces armées et de l'industrie.

Quoique Lehman ait accompli un tâche gigantesque, il est déjà bien dépassé. Son ouvrage intéressera surtout ceux qui s'occupent d'hygiène du travail et de santé industrielle.

C. R. Giroux

Pension Funds and Economic Power, by Paul Harbrecht, s.j., New York City: The Twentieth Century Fund, 41 East Seventieth Street, 21, 328 pp., 36 tableaux, 1 graphique, 2 appendices, $\$ 5.00$.

Le système de propriété des biens de production aux Etats-Unis est loin d'être celui que l'on imagine candidement. Déjà la propriété productive, entre les mains des corporations, domine totalement l'économie américaine. En 1955, la valeur aux livres de l'actif des corporations constituait $70 \%$ de toute la richesse de la nation. En 1956, la liste de paie des corporations en dehors de l'agriculture et des entreprises non-financières s'élevait à $60 \%$ des salaires payés dans le pays. Un quart des travailleurs salariés appartenaient à un fonds de pension. Or, les fonds de pension dans l'industrie privée, croissant au rythme de plus de $\$ 4$ milliards chaque année et s'élevant actuellement à plus de $\$ 33$ milliards aux Etats-Unis, sont une des forces les plus importantes dans l'évolution du capitalisme américain marqué déjà par une rapide institutionalisation de la propriété. Ils constituent une vaste agglomération de biens appartenant à personne et administrés par des sociétés de fiducie.

Le problème du développement de ces fonds de pension, de leur modalité et de leur influence sur l'économie est analysé objectivement dans les dix cha. pitres de Pension Funds and Economic
Power: l'origine des fonds de pension; la propriété en transition; ce que sont les fonds de pension; leurs objectifs; théorie et pratique; l'impact des politiques gouvernementales; les bénéficiaires devant les tribunaux; l'anatomie du contrôle; les fonds de pension en 1970; la société au delà de la propriété.

L'auteur n'a pas fait oeuvre d'imagination. Il base son étude sur des données factuelles et présente 36 tableaux statistiques. En appendice on trouve le texte du plan de fonds de pension de la General Motors ainsi qu'un spécimen d'entente avec une société de fiducie pour l'administration des plans de fonds de pension.

Ce qu'il y a de caractéristique dans cet ouvrage, c'est que l'auteur, qui possède une bonne formation en droit et en philosophie sociale, ne se contente pas de décrire les mécanismes de ces fonds de pension et leurs rapports avec la législation et les forces économiques. Son dernier chapitre, en particulier, avec celui où il expose l'évolution de la propriété, est de nature à ouvrir des horizons à tous ceux qui désirent voir dans quelle direction nous nous acheminons avec notre régime des biens de production en Amérique du Nord.

La société s'onganise de plus en plus avec des formes de propriété où œe ne sont plus les propriétaires (quand il $\mathbf{y}$ en a) qui ont une influence et prennent les décisions. Le pouvoir est sé paré de la propriété. Mais qui détient ce pouvoir? Qui conservera ce pouvoir? «Dans le domaine économique, les droits sont désormais rattachés aux hommes et non pas aux choses. Ceci a toujours été vrai dans le domaine politique. L'élément nouveau que la propriété privée introduit dans les systèmes d'organisation sociale est qu'elle crée un champ où le pouvoir provient d'une source indépendante du pouvoir politique... et maintenant que le contrôle sur les biens est devenu concentré dans de grands blocs, le pouvoir sur les hommes est encore devenu centralisé. Ce qui subsiste de l'époque ou le système de propriété était l'institution dominante dans notre société est la séparation des pouvoirs économiques et politiques... \$ Les fonds de pension avec leur capacité d'investissement de plus en plus grande (ne sontils pas un des plus gros acheteurs d'ac- 